potentiation of morpholino doxorubicin by human liver microsomes. Cancer Res 1992, 52, 4379-4384.

24. Coley HM, Twentyman PR, Workman P. 9-Alkylmorpholinyl anthracyclines in multidrug resistance. Eur 7 Cancer 1990, 26, 665-667.

25. Slevin $M L$, Joel P. Prolonged oral etoposide in small cell lung cancer. Ann Oncol 1993, 4, 529-532.

26. Millward MJ, Newell DR, Balmano K, et al. Clinical and pharmacokinetic study of BMY 40481 (etoposide phosphate). Proc Am Assoc Cancer Res 1992, 33, 529.

27. Whitesell L, Geselowitz D, Chavany C, et al. Stability, clearance and disposition of intraventricularly administered oligonucleotides: implications for therapeutic application within the nervous system. Proc Natl Acad Sci USA 1993, 90, 4665-4669.

28. Wilson JM. Vehicles for gene therapy. Nature 1993, 365, 691-692.

29. Jain RK. Therapeutic implications of tumour physiology. Curr Opin Oncol 1991, 3, 1105-1108.

30. Bagshawe KD. Towards generating cytotoxic agents at cancer sites. Brf Cancer 1989, 60, 275-281.

31. Powis G. Signalling targets for anticancer drug development. Trends Pharm Sci 1991, 3, 1105-1108.

32. Brunton VG, Workman P. Cell-signalling targets for antitumour drug development. Cancer Chemother Pharmac 1993, 32, 1-9.

33. Wiebe VJ, Osborne CK, McGuire WL, De Gregario MW. Identifi- cation of estrogenic tamoxifen-resistant human breast tumours. $\mathcal{F}$ Clin Oncol 1992, 10, 990-994.

34. Riley R, Workman P. DT-diaphorase and cancer chemotherapy. Biochem Pharmac 1992, 8, 1657-1669.

35. Newton P. Liquid chromatography-mass spectrometry: essential tool for drug research. $L G-G C \ln t, 8,706-714$.

36. Morrison JG, Bisset D, Stephens IF, et al. The isolation and identification of cis-diammine-chloroplatinum(II)-DNA adducts by anion exchange chromatography and inductively coupled plasma mass spectrometry. Int f Oncol 1993, 2, 33-37.

37. Stevens AN, Morris PG, Iles RA, Sheldon PW, Griffiths JR. 5 Fluoruacil metabolism monitored by in vivo ${ }^{19} \mathrm{~F}$ NMR. $\mathrm{Br} f$ Cancer 1984, 50, 113-117.

38. Hickman JA. Apoptosis induced by anticancer drugs. Cancer Metastasis Rev 1992, 11, 121-139.

39. Jackson RC. The kinetic properties of switch antimetabolites. $f$ Natl Cancer Inst 1993, 85, 539-545.

40. Reidenberberg MM. Trends in clinical pharmacokinetics. Clin Pharmacokinet 1993, 24, 1-9.

41. Abernathy DR. Presidential address: notes of the American Society for Clinical Pharmacology and Therapeutics. Clin Pharm Ther 1992, 51, 475-477.

Acknowledgements- The authors thank the Cancer Research Campaign for financial support, including a Life Fellowship to PW.

\title{
European School of Oncology
}

\section{First Euro-American Forum on Lung Cancer Treatment}

\author{
P.A. Bunn Jr., N. Van Zandwijk, U. Pastorino, J. Aisner, P. Alberto, \\ R. Arriagada, D. Carney, R. Cornis, C. Dittrich, U. Gatzemeier, R. Ginsberg, \\ F.A. Greco, H.H. Hansen, P. Harper, R. Henriksson, H. Huber, P. Klener, \\ T. LeChevalier, R. Lewensohn, N. Murray, N. Niederle, P. Postmus, \\ R. Rosell, G. Scagliotti, J.P. Sculier, T. Splinter, R. Stahel, M. Symann, \\ N. Thatcher, $M$. Tonato and $A$. Turrisi
}

\section{INTRODUCTION}

THE First Euro-American Forum on Lung Cancer Treatment, designed and organised by the European School of Oncology, was held in Vienna, Austria on 9-10 December 1993 with the aim of reviewing the current and future state of the art in lung cancer therapy. The workshop participants, listed above, represented the disciplines of prevention, surgery, radiotherapy and chemotherapy. There was guarded optimism expressed by the participants that recent combined modality therapies had made small improvements in survival. Advances in preventing second malignancies have also occurred. There was optimism that newly available chemoprevention strategies, chemothera- peutic agents, radiation and surgical techniques and biologically based therapies might lead to even further survival gains in the near future. Because most of the survival improvements have occurred in small increments, the need for large prospective randomised trials was emphasised. Rather than stressing pessimism toward lung cancer patients, we should encourage primary care physicians and related specialists to refer patients to specialists with appropriate experience in lung cancer so that optimal care can be delivered. By necessity, this care requires collaborative interactions among the specialities. 
THERAPEUTIC ISSUES IN LOCALISED NON-SMALL CELL LUNG CANCER (NSCLC)

Stage I NSCLC

Relapse for T1NO NSCLC after complete resection is approximately $20-30 \%$, and second primary tumours represent a frequent and significant problem. There are currently two large multi-centre randomised trials in Europe and North America on resected stage 1 patients with NSCLC. These trials evaluate retinol and $N$-acetyl cysteine or 13-cis retinoic acid, respectively. Further large-scale randomised trials with retinoids should await the result of these studies. In the meantime, small pilot studies testing new regimens and new prevention agents should be performed with intermediate biomarkers as relevant endpoints.

Within stage 1 disease, there are patients with a poorer prognosis which may be defined by new biological markers. These patients might benefit from adjuvant treatments. With reference to new biological markers of potential prognostic significance, it is recognised that there are several potential markers, such as blood group antigens, k-ras, NCAM, Ki67 and P53, which have been proposed to have prognostic significance in small retrospective studies. Clinical pathological correlations from large trials, using banked tissue blocks, collected on patients of known stage, outcome and treatment parameters are needed. The emphasis in these studies should be on evaluations of multiple markers on individual samples.

Resected stage II and IIIA (excluding mediastinoscopy positive N2 disease)

There is no accepted adjuvant therapy for completely resected stage II and IIIA disease which increases survival. There are randomised trials showing no survival benefit and others which suggest that there might be a small survival benefit from systemic therapy. Radiation therapy decreases local recurrence in completely resected patients with squamous cell carcinoma but does

Correspondence to U. Pastorino at the European School of Oncology, Via Ripemonti 66, 20141 Milan, Italy.

P.A. Bunn is at the University of Colorado Health Science Center, Denver, Colorado, U.S.A.; N. van Zandwijk is at the Netherlands Cancer Institute, Amsterdam, The Netherlands; J. Aisner is at the University of Maryland Cancer Center, Baltimore, Maryland, U.S.A.; $P$. Alberto is at the University Hospital, Geneva, Switzerland; $R$. Arriagada is at the Institut Gustave-Roussy, Villejuif, France; D. Carney is at the Mater Private Hospital, Dublin, Ireland; R. Cornis is at the Jefferson Cancer Center, Philadelphia, Pennsylvania, U.S.A.; C. Dittrich is at the University of Vienna, Vienna, Austria; U. Gatzemeier is at the Grosshansdorf Hospital, Grosshansdorf, Germany; R. Ginsberg is at the Memorial Sloan Kettering Cancer Center, New York, U.S.A.; F.A. Greco is at the Sarah Cannon (Minnie Pearl) Cancer Center, Nashville, Tennessee, U.S.A.; H.H. Hansen is at the Finsen Institute, Copenhagen, Denmark; P. Harper is at Guy's Hospital, London, U.K.; R. Henriksson is at the University of Umea, Umea, Sweden; H. Huber is at the Universitatsklinik fur Innere Medizin I, Vienna, Austria; $P$. Klener is at the Universita Karlova, Prague, Czech Republic; T. LeChevalier is at the Institut Gustave-Roussy, Villejuif, France; R. Lewensohn is at the Karolinska Hospital, Stockholm, Sweden; N. Murray is at the British Columbia Cancer Agency, Vancouver, Canada; N. Niederle is at the Zentrum fur Innere Medizin, Leverkusen, Germany; P. Postmus is at the Free University Hospital, Amsterdam, The Netherlands; R. Rosell is at the Hospital de Badalona Germans, Barcelona, Spain; G. Scagliotti is at the S. Luigi Gonzaga Hospital, Orbassano, Italy; J.P. Sculier is at the Institut J. Bordet, Brussels, Belgium; T. Splinter is at the University Hospital Dijkzigt, Rotterdam, The Netherlands; R. Stahel is at the University Hospital, Zurich, Switzerland; $M$. Symann is at the Universite Catholique de Louvain, Brussels, Belgium; N. Thatcher is at the Christie Hospital NHS Trust, Manchester, U.K.; M. Tonato is at the Policlinico Monteluce, Perugia, Italy; and A. Turrisi is at the University of Michigan, Michigan, U.S.A. Received 4 Mar. 1994; accepted 8 Mar. 1994. not improve survival. Since there is no universally accepted postoperative therapy, we support new randomised trials to better evaluate adjuvant therapy in this setting.

\section{'Unresectable' IIIA, IIIB disease}

There was agreement that surgery alone was not standard treatment for patients with bulky mediastinal disease or patients with N2 disease proven at mediastinoscopy. For unresectable stage III patients, combined modality approaches, including combination chemotherapy with local therapy (surgery or radiotherapy), have been shown to improve survival in randomised trials and meta-analyses of these trials. Cisplatin-containing regimens have been shown to improve survival when combined with surgery and/or radiation therapy. It is not clear that current combinations without cisplatin are effective.

The meta-analysis data show a positive survival effect with cisplatin-based combination used before chest radiotherapy or combined concurrently with thoracic radiation. The optimal schedule and combining radiotherapy with cisplatin need further study.

The standard dose of thoracic radiation therapy has been $60-65$ Gy which produces a 5-year survival of only $5 \%$. Therefore, there is room for investigation of dose escalation using once-a-day schemes, and exploration of altered fractionation in the hope of improving local control and survival. The volume of irradiation may include clinically uninvolved nodes. Although traditional at many centres, it is not clear that this is necessary and may increase morbidity, particularly with multi-modality strategies.

\section{Neoadjuvant studies with surgery after induction tharapy for unresectable patients}

There are two recent, small, randomised trials of chemotherapy before surgery versus surgery alone in operable stage IIIA NSCLC. These trials show very poor survival in the group identified with IIIA disease with surgery alone. For clinical and mediastinoscopy defined IIIA disease, chemotherapy alone or chemotherapy plus radiotherapy produces complete pathological remission in 10-30\%. Radiographical evaluations are not always predictive for response. Long-term survival is seen in some patients with complete response. The role of surgery after combined modality is feasible, with slightly increased morbidity; however, it is not clear whether surgery improves outcome as opposed to non-surgical management. There are two large randomised trials addressing these issues and others may be needed. The role of radiotherapy in induction is advocated by some, but remains investigational.

\section{New agents}

At this time, there are a number of promising new chemotherapeutic agents discussed in the next section. However, prior to integration into induction regimens, these will require further study to define their toxicity and efficacy. These agents are likely to require integration early in therapy if they are to improve outcomes.

\section{CHEMOTHERAPY ISSUES IN METASTATIC (STAGE IV) NSCLC}

Although metastatic (stage IV) NSCLC is uniformly fatal, effective palliation is a major goal. Supportive and symptomatic care should be offered to these patients by a physician who is experienced in palliative care issues. This includes the judicious use of radiotherapy and chemotherapy, which often improve quality and quantity of life. 
Chemotherapy prolongs the survival of stage IIIB and IV NSCLC patients who have a good performance status. This has been established scientifically by recent meta-analyses of worldwide prospectively randomised studies of chemotherapy versus best supportive care. The magnitude of the benefit is modest, with a prolongation of survival of about 2 months at the median and nearly a doubling of 1 -year survival. However, by 2 years, the vast majority of patients have died from progressive lung cancer, despite the chemotherapy. There are no data to show that the appropriate use of anti-tumour therapy increases the overall cost of caring for these patients, and a Canadian study suggested that chemotherapy could lower overall costs. Because the data in this respect are sparse, additional study of cost-benefit issues is warranted.

Combination chemotherapy regimens containing cisplatin significantly prolonged survival in the meta-analyses of these studies. The optimal number of drugs, dose, schedule and duration of therapy are undefined, although there is no evidence that dose intensification improves outcome.

There is no standard vehicle for measuring quality of life and improvement of symptoms, and this remains an important but elusive goal. Future studies should incorporate these analyses into the study design.

Because no chemotherapeutic agent produces a response rate higher than $25 \%$, nor cures stage IV NSCLC patients, efforts should focus on finding and evaluating new agents. Because the response rate to chemotherapy is related to performance status and amount of prior weight loss, chemotherapy for stage IV NSCLC patients should be offered preferentially to those with good performance status and minimal prior weight loss.

CPT-11, docetaxel, gemcitabine, paclitaxel and vinorelbine have anti-tumour activity in NSCLC. Early data suggest that topotecan may also have activity. In two prospective, randomised trials from the U.S.A. and from Europe, vinorelbine alone and combined with cisplatin was shown to prolong survival in stage IV NSCLC.

Pilot studies of the other new agents with novel mechanisms of action used alone and as part of combinations are warranted to develop combination regimens for phase III comparisons to commonly used cisplatin regimens.

Response rates to chemotherapy are consistently higher in stage III than stage IV NSCLC. Therefore, clinical trials should stage patients appropriately and should distinguish between stages IIIA, IIIB and IV. Since the response rates to chemotherapy are higher in stage III than stage IV NSCLC, and since chemotherapy appears to have a greater effect on survival in stage III, effective new combinations should be evaluated in stage III disease as soon as their efficacy is proven in stage IV. There is currently no evidence that dose-intensive therapy or routine growth factor support are beneficial for patients with NSCLC.

\section{CHEMOTHERAPY ISSUES IN LIMITED AND EXTENSTVE STAGE SCLC}

At present, patients with both limited and extensive disease stage SCLC at diagnosis are considered candidates for systemic combination chemotherapy as primary treatment because it significantly prolongs survival. SCLCs are highly sensitive to chemotherapy and radiotherapy, so that remissions of up to $90-95 \%$ are possible in the limited stage. Combination chemotherapy with two-, three- or four-drug combinations are unequivocally superior to monotherapy in patients with good performance status. The most commonly used regimens are
PE (cisplatin/etoposide), CAV (cyclophosphamide, doxorubicin and vincristine) and alternating $\mathrm{CAV} / \mathrm{PE}$ and $\mathrm{CAE}$ (cyclophosphamide, doxorubicin and etoposide).

Two randomised trials by Roth (U.S.A.) and Fukuoka (Japan) showed no significant differences in survival between $P E$ and CAV and alternating CAV/PE. However, PE was less myelosuppressive in these studies. In a randomised study of the Hellenic Oncology Group, cisplatin was replaced by carboplatin and the carboplatin/etoposide combination demonstrated similar activity to $\mathrm{PE}$, but with less toxic non-haematological sideeffects. The dosage of cisplatin or carboplatin was in the range of $80-100 \mathrm{mg} / \mathrm{m}^{2}$ or $300-400 \mathrm{mg} / \mathrm{m}^{2}$, respectively. A randomised study by the National Cancer Institute (U.S.A.) showed no advantage for increasing the etoposide and cisplatin dose. However, a recent French randomised trial showed a significant positive effect for a higher initial dose of cisplatin and cyclophosphamide. Etoposide dosage that was most often administered per course was $100 \mathrm{mg} / \mathrm{m}^{2}$ days $1-3$ or $50 \mathrm{mg} / \mathrm{m}^{2}$ days $1-5$. New combination treatments should be compared to a "standard" regimen.

The rate of complete remission which can be obtained is $20-50 \%$, with an overall response rate of $90-95 \%$ for patients with limited stage disease, and $80 \%$ with $10-20 \%$ complete responses in extensive stages. The median survival time is $14-18$ months for limited stage and 9-12 months for extended stage. In many series, the 3 -year survival ("long-term survival") is $10-30 \%$ of patients with limited stage disease. Five-year survival rates are $10-30 \%$ for limited stage and $1 \%$ for extensive stage disease. After 3 years, the rate of developing second primary cancers exceeds the rate of relapse. Because the risk of second tumours is extremely high in long-term survivors (actuarial risk $>50 \%$ at 5 years) and because this risk is higher in those who continue to smoke, these patients should be encouraged to discontinue smoking, and randomised studies of chemopreventive agents should be instituted. There are no such randomised trials in progress in the U.S.A. or Europe to our knowledge.

Currently, there is no evidence that dose-intensive therapy produces superior results compared to standard doses. There are ongoing trials in the U.S.A. and Europe which compare high-dose chemotherapy in combination with growth factors to standard treatment. Until the final results of those trials are available, there is no evidence that growth factor support is routinely needed. A prospective, randomised trial conducted by the Southwest Oncology Group (SWOG) showed increased thrombocytopenia and other toxicities when granulocytemacrophage colony-stimulating factor (GM-CSF) was given concurrently with the thoracic irradiation. A trial from Wayne State showed a similar problem with G-CSF and concurrent thoracic irradiation in NSCLC. Therefore, the concurrent use of protracted large volume radiotherapy and concurrent growth factor should be avoided. Since there is no evidence that intensive therapy with autologous bone marrow/peripheral stem cell support prolongs survival, its use should also be considered experimental.

Weekly chemotherapy administration produced excellent phase II results, but two phase III trials in Europe showed negative results. Murray and co-workers developed a more intensive weekly regimen, termed CODE (cyclophosphamide, vincristine, doxorubicin and etoposide) and reported excellent results in a phase II study. This approach seems appropriate for further trials for patients with good performance status. A trial of CODE versus CAV/PE is ongoing in the U.S.A. and Canada.

The duration of the initial chemotherapy averages four to six 
cycles. Maintenance chemotherapy does not afford any clinically relevant or significant survival advantage. Depending on the situation at relapse or progress, chemotherapy and/or radiotherapy should be considered.

For the near future, it needs to be established whether the addition of any drug to standard therapy increases the efficacy. In addition, the question of whether high-dose chemotherapy in combination with growth factors and/or stem cell support improves survival has to be proven in comparison to standard chemotherapy doses.

Several new agents showed evidence of activity in SCLC, including CPT-11, docetaxel, gemcitabine, paclitaxel and topotecan. Their use in combination should proceed rapidly so that phase III trials can be instituted promptly if results are promising.

New therapies based on biological principles, such as antigrowth factors, anti-metastatic agents, conjugated antibodies, etc., warrant prompt phase I study. Appropriate end-points and study design are essential for optimal evaluation of the efficacy of these agents.

\section{COMBINED CHEMORADIOTHERAPY IN LIMITED SCLC: RADIOTHERAPY ISSUES}

Two meta-analyses (one using individual data) of randomised trials, comparing the addition of thoracic radiotherapy to combination chemotherapy, found a survival advantage with the use of thoracic radiotherapy. The survival advantage approximates $5 \%$ at 5 years. These trials were predominantly cyclophosphamide and/or doxorubicin based. These trials demonstrated approximately a 3-fold decrease in local failure, but added toxicity when radiotherapy was added. It is conceivable that the use of other active compounds, such as podophyllotoxines and platinum compounds, may reduce the toxicity of the combined approach. An analysis from a French trial, using alternating chemotherapy and radiotherapy, suggested that local failure predominates when the first site of failure is analysed within a competing risk approach. Despite these data, many continue to believe that systemic failure remains the most important issue, and local control provided by radiotherapy is a secondary issue. The optimal method of integration of chest radiotherapy with chemotherapy cannot be extracted from these data. The majority of the group favoured the addition of thoracic radiotherapy in limited stage patients, but some dissent about the universality of this application was registered. Some felt that routine large volume thoracic radiotherapy (TRT), is not always necessary, particularly for elderly or infirm patients with poor performance status.
Optimal radiotherapy factors remain poorly defined and worthwhile subjects of future research. Local control and local failure seem to be reasonable markers to track the effect of local therapy. Improvement in survival remains a primary target of therapy. Doses in the range of 45-50 Gy remain common, and, to date, higher doses seem appealing but of unproven merit. The target volume varies from centre to centre. Some include contralateral hilar and supraclavicular lymphatic regions even when clinically negative. For delayed concurrent, sequential and alternating methods, reducing the volume as the chemotherapy reduces the tumour size may be a reasonable strategy, but some investigators continue to press for larger volumes which maintain the original boundaries of the tumour. There was consensus to define the target volume and to report the dose it received. The use of posterior spinal cord shields and the potential to reduce target volume dose make this an unappealing technique. Fractionation studies continue. There is room for more trials exploring optimal fractionation or duration of combined modality therapy. Timing of the TRT, early or late, produced the question of definition of terms. A randomised study of the National Cancer Institute of Canada showed that early radiotherapy administration with cycle two was better than delaying to cycle six. However, a CALGB trial suggested that early TRT with cycle 1 produced chemotherapy dose reductions and poorer median and 2-year survival than when the TRT was given with cycle four. Several randomised trials showed no benefit to giving TRT at the completion of all chemotherapy. Sequential treatment with delaying administration of TRT until completion of four, six and eight cycles had few advocates. However, there is clearly room for information about this method in comparison to earlier TRT.

Current worldwide efforts have largely adopted either concurrent or alternating strategies, with sequential treatments being infrequently used. Although favoured by some participants, sequential treatment has not been favoured in current North American trials. A comparison between early alternating radiotherapy treatment and late sequential radiation treatment is the subject of several trials in Europe.

Trials isolating TRT variables of dose, volume, fractionation, timing and sequence continue to be of interest since these factors remain undefined at this writing.

All agreed that survival differences in all randomised trials reported to date were small, thus future, prospective, randomised trials should be of appropriate size to have the power to detect small differences.

Acknowledgement-The Forum was supported by an unrestricted educational grant from Bristol-Myers Squibb Company. 